\title{
(C) OPEN ACCESS \\ Paediatric chest wall trauma causing delayed presentation of ventricular arrhythmia
}

\author{
Angela M Tegethoff, Emerald Raney, Jenny Mendelson, Michael R Minckler
}

Department of Emergency Medicine, Banner-University Medical Center Tucson, Tucson, Arizona, USA

\section{Correspondence to} Angela M Tegethoff, tegethof@ email.arizona.edu

Accepted 21 June 2017

\section{SUMMARY}

This report describes a paediatric patient presenting with haemodynamically stable non-sustained ventricular tachycardia 1 day after minor blunt chest trauma. Initial laboratory studies, chest X-ray and echocardiography were normal; however, cardiac MRI revealed precordial haematoma, myocardial contusion and small pericardial effusion. Throughout her hospital course, she remained asymptomatic aside from frequent couplets and triplets of premature ventricular contractions. Ectopy was controlled with oral verapamil. This case highlights how significant cardiac injury may be missed with standard diagnostic algorithms.

\section{BACKGROUND}

This is a rare paediatric case of blunt myocardial injury caused by a relatively benign mechanism of injury resulting in a serious rhythm abnormality. Additionally, the delayed presentation of symptoms with negative biomarkers and transthoracic echocardiography (TTE) makes this a rare presentation of blunt myocardial injury.

\section{CASE PRESENTATION}

An 8-year-old girl presented to the emergency department (ED) with reported ventricular tachycardia. The patient stated that when she stood up to walk out of the classroom she began to experience lightheadedness and had two presyncopal episodes. She then went to her school's nurse's office where she was found to have intermittent episodes of faint and rapid pulse, so emergency medical services were called. On scene, rhythm strip showed a wide rapid rhythm consistent with ventricular tachycardia so she was brought to the ED for further evaluation. Her Corrected QT Interval (QTc)on the first ECG obtained was 423. In the ED, she denied chest pain, shortness of breath or palpitations and remained haemodynamically stable. She was noted to have multiple runs of stable non-sustained ventricular tachycardia with the longest run lasting approximately $10 \mathrm{~s}$. Intravenous access was established and she was given a normal saline bolus. She was urgently evaluated by paediatric cardiology and paediatric electrophysiology. Her ECG showed frequent ventricular ectopy but were otherwise unremarkable.

Her laboratory tests including complete blood count, comprehensive metabolic panel, troponin I and chest radiograph were unremarkable. Approximately 1 hour into her hospital course her mother arrived and provided further history. She stated that 24 hours prior to arrival in the ED, the patient was riding a non-motorised scooter around her front yard. The front wheel caught on a lip of the cement and she ran into the garage door, with handle bars turned such that one end of the handle bar contacted the garage door while the other end struck her chest wall. She continued playing and experienced no symptoms other than some mild localised chest wall pain. There was no overlying chest wall deformity, bruising, ecchymosis or haematoma aside from a small left parasternal abrasion noted on physical exam. She was admitted to the paediatric intensive care unit (PICU), echocardiogram was obtained and was unremarkable. On hospital day 2 , her chest MRI showed a right ventricular cardiac contusion, subacute precordial haematoma and a small pericardial effusion with possible pericardial haematoma. She remained in the hospital for 3 days for continued monitoring due to recurrent runs of preventricular contractions.

\section{INVESTIGATIONS}

- ECG 12 lead

- Portable upright A/P one view chest radiograph

- Transthoracic echocardiogram

- Cardiac MRI with and without contrast with flow velocity mapping

- Complete blood count, comprehensive metabolic panel, troponin I, brain natriuretic peptide, thyroid-stimulating hormone reflex T4, phosphorus, magnesium, erythrocyte sedimentation rate, $\mathrm{C}$ reactive protein, venous blood gases, drug screen, urinalysis

\section{DIFFERENTIAL DIAGNOSIS}

Arrhythmia is a rare presenting complaint in the paediatric ED, occurring in only 55 of every 100000 patients. Sinus tachycardia and supraventricular tachycardia are the most common arrhythmia in children. ${ }^{1}$ The usual presenting symptoms are palpitations, fatigue and/or syncope. Although rare in children, ventricular tachycardia must be considered because it is a known cause of sudden death in paediatrics.

One of the common causes of ventricular tachycardia in children is myocarditis. Usually the aetiology of myocarditis is viral, classically Coxsackie B. The presenting symptoms can include: tachypnoea, chest pain, syncope and palpitations. ${ }^{2}$ Ventricular 
arrhythmias may also be the initial manifestation. A study by Vignola et al found $26 \%$ of patients referred for cardiac evaluation had an unknown cause of ventricular arrhythmias. Of these patients who underwent biopsy, half had an underlying myocarditis that was clinically silent. ${ }^{3}$ An echocardiogram is used to look for structural changes associated with myocarditis such as dilated cardiomyopathy and reduced ejection fraction.

Another known cause of arrhythmias is electrolyte disturbances. Cardiac contractility depends on intracellular and extracellular shifts of ion gradients, and alterations in these gradients can lead to abnormalities in conduction. Abnormal potassium levels are a well-known ionic cause of arrhythmias. Hypokalaemia can be caused by conditions including urinary and gastrointestinal losses. ${ }^{4} \mathrm{ECG}$ manifestations are decreased T-wave amplitude, ST segment depression and development of $U$ waves. Patients are at an increased risk of torsades de pointes or ventricular tachycardia because of the increased duration of the action potential and refractory period induced by hypokalaemia. ${ }^{4}$ Hyperkalaemia causes a shortening of the action potential and slowed conduction. Hyperkalaemia ECG changes include peaked T waves, widened QRS complexes and PR prolongation. ${ }^{5}$ It has been associated with sinus bradycardia and eventual sinus arrest or ventricular tachycardia and ventricular fibrillation.

Initial laboratory workup helps rule out or identify the cause of an arrhythmia. This workup should include: a toxicology screen, blood culture, viral panel, cardiac enzymes and serum electrolytes. Echocardiograms are done to look for structural abnormalities, potential tumours or cardiomyopathies, ${ }^{1}$ which are also known to cause arrhythmias. These initial tests in our patient came back normal which eliminated most of the common causes of ventricular tachycardia.

When it was later learnt that our patient had sustained blunt trauma to her chest, this was suspected to be the source of her symptoms. Arrhythmias are a complications of blunt cardiac injury, and they typically manifest as sinus tachycardia, atrial fibrillation or ventricular or atrial premature contractions. ${ }^{6}$ With blunt trauma there is also potential for pericardial effusion, rupture of the myocardium or rupture of the valves. Patients with a history of blunt myocardial injury who develop ECG changes, a new murmur or chest pain should be evaluated with cardiac enzymes and echocardiograph to help identify structural injuries.

\section{TREATMENT}

- Verapamil $40 \mathrm{mg}$ three times a day, increased to $60 \mathrm{mg}$ three times a day.

\section{OUTCOME AND FOLLOW-UP}

On hospital day 1, CXR and TTE were performed. The echocardiogram results showed no acute pathology. After being evaluated by paediatric cardiology she was admitted to the PICU. On hospital day 2, cardiac MRI was obtained that revealed right ventricular contusion (correlating with origin of ventricular ectopy) and oedema with regional wall motion abnormalities involving the right ventricular outflow tract and free wall. The MRI also showed a subacute precordial haematoma extending from the level of the great vessels across the left and anterior border of the heart, anterior to the pulmonary artery and right ventricular outflow tract and a small pericardial effusion with possible pericardial haematoma. She was started on verapamil $40 \mathrm{mg}$ every 8 hours on arrival; on hospital day 3 her dose was increased to $60 \mathrm{mg}$ every 8 hours due to sustained frequent ectopic beats. On day 4, she continued to have premature ventricular contractions (PVCs) but with a decrease in frequency. The paediatric cardiology electrophysiologist evaluated the patient at bedside and cleared her for discharge. She was instructed to continue verapamil at home, to follow-up with paediatric cardiology in 2 weeks and a Holter monitor was ordered. Additionally, she was advised to avoid vigorous physical activity for 4 weeks. One month after discharge, the patient presented to the ED with non-specific leg weakness, spasms, fatigue and complaints of 'feeling hot all over'. The emergency providers attributed her symptoms to her verapamil therapy but wanted to rule out electrolyte/metabolic derangements. She was placed on cardiac monitoring which revealed her continuing PVCs. Patient remained haemodynamically stable. Basic metabolic panel, complete blood count, magnesium, phosphorus, venous blood gas and chest radiography were unremarkable. She was discharged home with recommended outpatient cardiology follow-up. One month later, she was seen by her outpatient cardiologist. In the interim from her original hospital stay aside from the ED visit, she experienced no shortness of breath chest pain, diaphoresis, palpitations, dizziness or syncope. She was restricted from physical education class for approximately 1 month, following the accident. She was otherwise able to keep up with her peers without difficulty. After her month of restricted activity, she was allowed to return to full physical activity. Her mother was reassured that she should be treated as a normal healthy child without any restrictions and that no pre-procedural bacterial endocarditis prophylaxis would be necessary. She has continued to be followed by paediatric cardiology every 6 months with ECG and 24 hours Holter monitoring prior to each appointment. Her last two Holter recordings demonstrated 26\%-27\% PVCs. Her ECGs have continued to demonstrate sinus rhythm with monomorphic PVCs and occasional couplets. Her QTcs on her last four ECGs were 406, 401, 423 and 441.

\section{Box 1 Eastern Association for the Surgery of Trauma} guidelines for evaluation and treatment of myocardial contusion $^{10}$

Level I

Admission ECG should be obtained in all patients where there is suspected Blunt Cardiac Injury.

Level II

1. If admission ECG is abnormal, the patient should be admitted for continuous ECG monitoring for 24-48 hours. If admission ECG is normal, further pursuit of diagnosis should be abandoned.

2. If the patient is haemodynamically unstable, an imaging study such as transthoracic echocardiogram or transesophageal echocardiogramshould be obtained.

3. Nuclear medicine scans add little compared with echocardiography and are not useful if echocardiography has been performed.

\section{Level III}

1. Elderly patients with known cardiac disease, unstable patients and those with abnormal admission ECGs can be safely operated on provided that they are closely monitored.

2. The presence of a sternal fracture does not predict the presence of $\mathrm{BCl}$ and does not necessarily indicate that monitoring should be performed.

3. Neither creatine phosphokinase analysis nor measurement of circulating cardiac troponin T are useful in predicting which patients have or will have complication related to $\mathrm{BCl}$. 


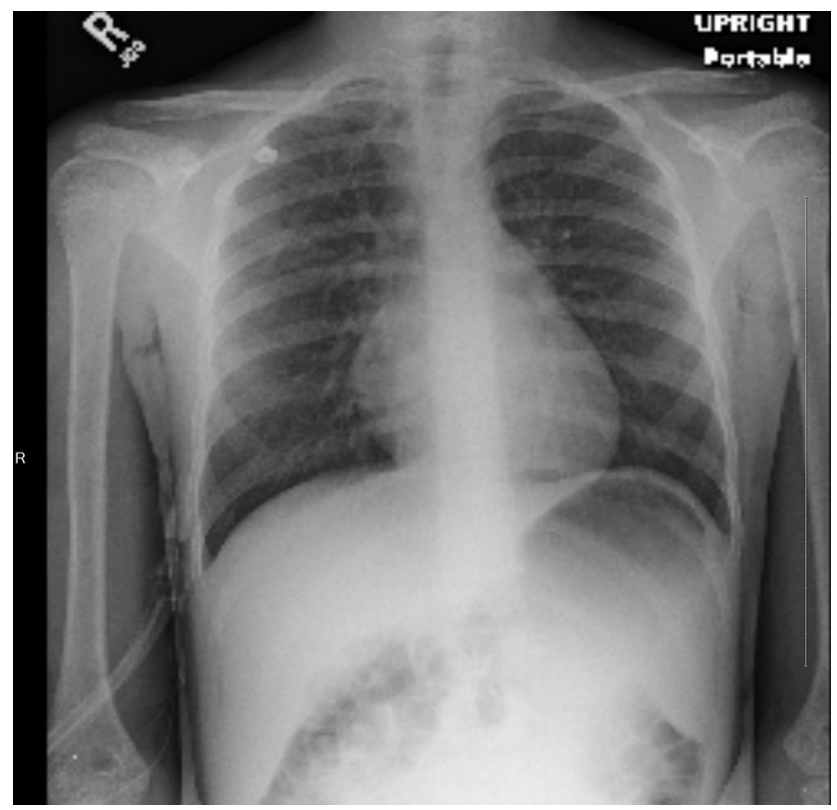

Figure 1 Portable frontal upright chest. The heart is normal in size. The lungs are clear. There is no pleural effusion or pneumothorax. The visualised osseous structures are within normal limits. No acute cardiopulmonary disease.

Follow-up exercise treadmill after her most recent cardiology appointment demonstrated appropriate suppression of PVCs during exercise. She remained on verapamil $120 \mathrm{mg}$ total daily therapy for 1 year following the inciting event;however, due to her continuing frequent PVCs her verapamil was discontinued and metoprolol $25 \mathrm{mg}$ extended release tablets taken at bedtime were started. She did not have further cardiac MRI, echocardiogram, formal cardiac electrophysiology or genetic workup for specific cardiac abnormalities although this may be important to consider in further investigations. She will continue follow-up every 6 months for the time being.

Of note, there was a discrepancy in the terminology used by the emergency medicine physicians and the paediatric cardiologists to label the rhythm abnormality seen in this case. The ED physicians felt that stable ventricular tachycardia was an appropriate description of the presenting rhythm abnormality, given it was a wide monomorphic tachycardia. Whereas, because the patient was haemodynamically stable, paediatric cardiology described it as a monomorphic accelerated ventricular rhythm of left bundle branch morphology. Another important differentiation when a patient presents with tachycardia is sustained versus non-sustained. Non-sustained ventricular tachycardia is defined as a run lasting less than $30 \mathrm{~s}$ of three or more consecutive premature ventricular contractions which spontaneously resolve. Non-sustained ventricular tachycardia typically does not require treatment and is usually the result of a re-entry circuit. Sustained ventricular tachycardia however, is considered a medical emergency requiring immediate cardioversion. It is defined as consecutive ectopic ventricular beats for more than 30 s, usually arising from coronary artery disease.

\section{DISCUSSION}

Trauma is the leading cause of death in individuals under 18 years of age. Although thoracic trauma only accounts for a small percentage of admissions to a trauma centre, it is second only to head injury as the most common cause of death in this age group. Blunt myocardial injuries range from asymptomatic

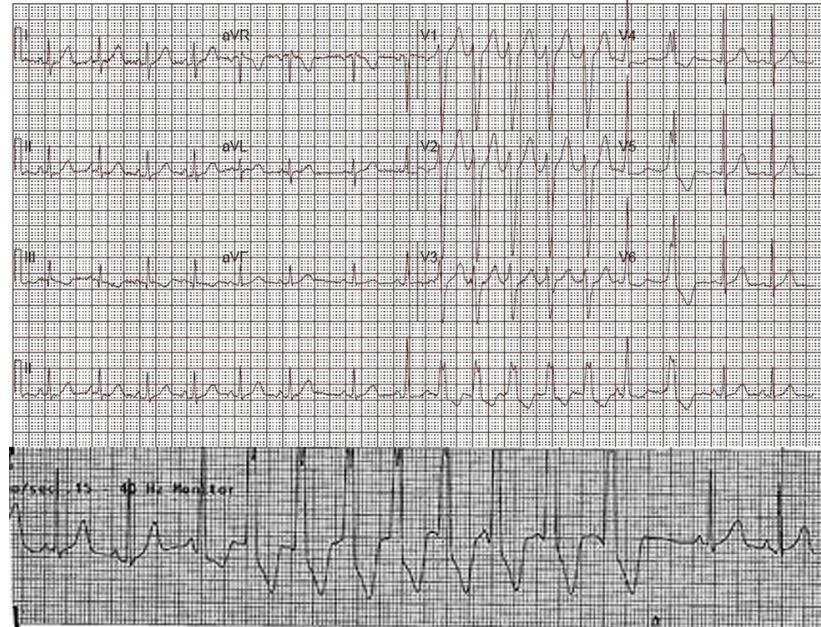

Figure 2 ECG. Monomorphic accelerated ventricular rhythm of LBBB morphology.

myocardial contusion to catastrophic myocardial rupture. Myocardial contusion is the most common and most easily overlooked injury. This is in part due to the lack of consistent correlation with cardiac biomarkers, ECG, echocardiograms and other diagnostic imaging modalities. ${ }^{2}$ The most common causes of blunt myocardial injury are: motor vehicle accidents, pedestrian struck, falls from a height of greater than 20 feet, crush injuries and abuse. ${ }^{8}$ This case demonstrates several important learning points including the mechanism of injury, clinical presentation and diagnostic methods for blunt cardiac injury.

Cardiac contusion is often overlooked because it is difficult to detect with standard diagnostic techniques and because it is overwhelmingly benign and often goes unnoticed by the patient. It is likely that cardiac contusion is a far more common injury than data suggest simply due to the fact that if the injury is not sustained in the setting of a high-impact multisystem trauma, the patient may never present for workup at all. Given the anatomic location of the heart, it is generally well protected behind the sternum and rib cage. Therefore, the more common scenario of symptomatic blunt cardiac injury is one of significant forces delivered to the thorax resulting in immediate myocardial rupture with instant death or immediately apparent pathology. Data on the epidemiology of paediatric blunt cardiac injury are limited. However in one large epidemiological study of paediatric cardiac trauma reported on 184 cases of blunt cardiac injury in children at 16 participating centres. In this study, the median age was 7.4 years and $73 \%$ were male. Myocardial contusions accounted for $95 \%$ of the diagnoses with the leading mechanism of injury being motor vehicle collision versus pedestrian $(39.7 \%)$ or passenger $(31.0 \%)$. The significant majority $(95 \%)$ had suffered multisystem trauma $(60 \%$ with evidence of external trauma) with pulmonary contusions $(50.5 \%)$ and rib fractures (23.0\%) being the most common associated internal injuries. ${ }^{8}$ Our case is extremely rare because the mechanism of injury was low impact and unassociated with external evidence of thoracic trauma or trauma to other systems, and the patient presented a day after the injury but with very serious cardiac pathology.

In addition to the atypical mechanism and delayed presentation, it is interesting that the current recommended diagnostic modalities for detecting blunt cardiac injury did not reveal pathology in this case. Specifically, our patient had an abnormal admission ECG but unremarkable TTE and cardiac enzymes. The Eastern Association for the Surgery of Trauma (EAST) has 
generated guidelines for the evaluation and treatment of myocardial contusion (box 1). These guidelines as well as several other techniques commonly used to screen for blunt cardiac injury will now be discussed.

Chest radiography, although not included in the EAST guidelines, is an inexpensive and rapid diagnostic modality that is obtained in most trauma situations. However, cardiac injury can only be inferred using this modality (unless obvious foreign bodies are seen) from evidence of significant intrathoracic trauma by the presence of haemothorax, rib fractures, intrathoracic free air or enlarged cardiac silhouette (although non-specific). ${ }^{9}$ Our patient's portable frontal upright chest radiograph (figure 1) revealed no acute cardiopulmonary disease.

ECG on arrival to the ED is now considered to be the most important screening test according to EAST. ${ }^{9} 10$ The ECG correlates with the development of cardiac complications with a sensitivity and specificity of $96 \%$ and $47 \%$, respectively. ${ }^{9} 1112$ If the patient is haemodynamically stable and the ECG is normal, the risk of having blunt cardiac injury requiring treatment is extremely unlikely, and therefore further workup or admission is not recommended. ${ }^{913-15}$ However, if the patient has an abnormal ECG, that patient should be admitted for monitoring. ${ }^{14}$ In our patient's case, her ECG revealed monomorphic accelerated ventricular rhythm of LBBB morphology (figure 2A,B) and she was therefore correctly admitted for monitoring.

Cardiac enzymes are sensitive markers for cardiac injury; however, as seen in our patient's case they may not always be reliable and are certainly not sufficient to rule in or out cardiac injury. It is possible that due to our patient's delayed presentation that her cardiac enzymes had already begun to fall. Radionuclide imaging is believed by EAST to add little given the availability of echocardiography. According to the EAST guidelines, echocardiography is only appropriate in the face of haemodynamic instability. The Dowd and Krug study ${ }^{8}$ found that in paediatric blunt trauma patients, there was only minimal correlation between echocardiography and abnormal ECG findings. In our case, however, it is interesting that such a sensitive test as echocardiography revealed no abnormalities, including no wall motion abnormalities when cardiac MRI did demonstrate this.

According to these guidelines based on our patient's abnormal admission ECG, it was appropriate to admit her for continuous cardiac monitoring for $24-48$ hours. In addition, since she was briefly considered haemodynamically unstable due to her presyncopal episodes, it was also appropriate to obtain a TTE. Where our case begins to deviate from the current guidelines is that even though the TTE was unrevealing, in the setting of the patient's serious ventricular rhythm abnormality, the decision was made to proceed with cardiac MRI. Cardiac MRI (figure 3A-C) revealed pathology including: precordial haematoma, myocardial contusion involving the right ventricular free wall with focal areas of delayed enhancement, significant regional wall motion abnormalities involving the right ventricular outflow tract and right ventricular free wall, with a small pericardial effusion. It is very rare for a trauma patient with suspected blunt cardiac injury to need an extensive investigation, including cardiac MRI, to make a diagnosis. One might think that since cardiac MRI was necessary to diagnose cardiac contusion in this case that the myocardial injury was minor, however, the MRI revealed gross and serious pathology. If the EAST guidelines had been strictly followed in our patient, it is possible that even a TTE would not have been considered and the workup certainly would not have proceeded to MRI. This may demonstrate the need to consider the adequacy of the current screening guidelines. Furthermore, although TTE has the advantage of being point of care and not

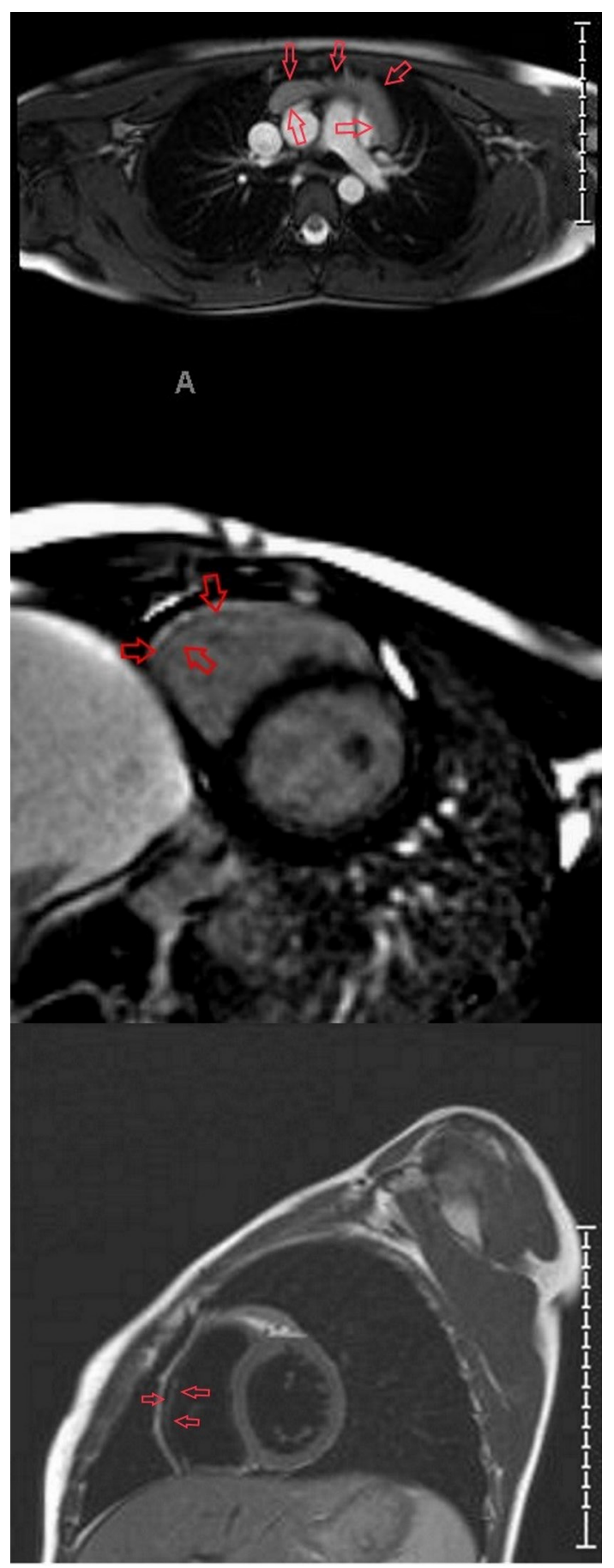

Figure 3 Cardiac MRI. Evidence of myocardial contusion involving the right ventricular free wall with focal areas of delayed enhancement, evidence of oedema (figure $3 \mathrm{C}$ ), significant regional wall motion abnormalities involving the right ventricular outflow tract and right ventricular free wall (figure 3B). Normal left ventricular size and function, LVEF of $69 \%$; small pericardial effusion with possible pericardial stranding/haematoma (figure 3A); normal right ventricular size with mildly depressed global function and regional wall motion abnormalities as described above. RVEF is $41 \%$. LVEF, Left ventricular ejection fraction; RVEF, right ventricular ejection fraction. Figure $3 \mathrm{~A}, 3 \mathrm{~B}$ and $3 \mathrm{C}$ follow in order. 
requiring patient transportation, Bock and colleagues found that TTE was not as sensitive at predicting major adverse cardiac events as cardiac MRI (CMR), as well as described poor correlation between TTE and CMR. ${ }^{16}$

To conclude, it is crucial to have a high index of suspicion for serious cardiac pathology even in low impact mechanisms with delayed presentations. Though cardiac MRI may not be feasible in many practice settings, it may represent a more accurate diagnostic modality when a negative cardiac workup is complicated by persistent episodic cardiac-rhythm abnormalities. It is reasonable to pursue cardiac MRI if there is high clinical suspicion of a significant underlying injury due to evidence of grossly abnormal electrical activity with otherwise negative workup (TTE and cardiac enzymes) as seen in our patient. It is possible that the current screening guidelines may be inadequate for detecting myocardial contusions. It is unclear however if more minor contusions go undiagnosed if it will significantly impact the patient's outcome and long-term health status. In the Dowd and Krug study, ${ }^{8}$ out of the 184 cases, 159 patients survived $(86.4 \%)$ and only 8 (5\% of survivors) had significant cardiac sequelae, most commonly mitral or tricuspid insufficiency or ventricular septal defect. During our literature review, we have not found any evidence of an initial arrhythmia secondary to blunt cardiac trauma progressing to ventricular fibrillation or ventricular tachycardia arrest, the chance of Vfib/Vtach arrest if not induced in the immediate setting is exceedingly rare. Specifically, in the Bock study, it was found that among 65 patients who were haemodynamically stable with normal screening ECG on arrival to the ED, there was not a single malignant arrhythmia or case of pump failure observed. ${ }^{16}$ Among the patients who developed myocardial contusion or segmental wall motion abnormalities, the majority of arrhythmia or hypokinesia resolved within 24 hours. Therefore, cardiac sequelae 1 year after cardiac contusion in haemodynamically stable patients are rare. ${ }^{16}$ In patients with a negative troponin I and minimal electrical disturbance prognosis is good. However, in more pronounced electrical disturbance, such as what our patient had, this indicates more severe underlying injury. There are case reports of blunt myocardial injury with no overlying chest wall trauma leading to death

Learning points

- Without a standard algorithm for diagnosis and treatment, complications of blunt myocardial injury can be overlooked and become fatal.

- Further diagnostic testing might be necessary in a paediatric case of seemingly insignificant myocardial blunt trauma. Negative laboratory results should not rule out potential injury. Every patient with blunt chest injury should be given strict return precautions regarding warning signs and symptoms. Additionally, a thorough follow-up plan should be established.

- The presentation of serious rhythm abnormalities can be delayed.

- Further research may be warranted in diagnosis of blunt myocardial injury. because the haemopericardium progressed to tamponade, so there is indeed a risk of death with this. ${ }^{1718}$

Further research is necessary to delineate a more reliable and cost-effective diagnostic algorithm for blunt myocardial injury.

Contributors AT: corresponding author, performed literature review, coordinated with coauthors to synthesise final data collection includinglaboratory values, ECGs, cardiac MRI and chest radiographs. Authored 'discussion, summary, background, investigations, treatment, portion of outcome/follow upand learning points/take home messages', wrote captions for images, citedreferences, edited and submitted final manuscript. ER: second author, collectedchest radiographs and cardiac MRI, reviewed these images with radiology facultyand formatted images with arrows pointing to pathology important to our case,authored portions of the 'case presentation, differential diagnosis andoutcome/follow up' for the manuscript, contributed to literature review, datacollection and citing references. JM: guarantor, reviewed, edited and providedfeedback on final manuscript. MRM: guarantor, provided mentorship throughoutauthoring process, collected data including laboratory values andelectrocardiogram tracings, collected articles for literature review, editedand provided feedback on manuscript drafts and final version.

Competing interests None declared.

\section{Patient consent Obtained.}

Provenance and peer review Not commissioned; externally peer reviewed.

Open Access This is an Open Access article distributed in accordance with the Creative Commons Attribution Non Commercial (CC BY-NC 4.0) license, which permits others to distribute, remix, adapt, build upon this work non-commercially, and license their derivative works on different terms, provided the original work is properly cited and the use is non-commercial. See: http://creativecommons.org/ licenses/by-nc/4.0/

(C) BMJ Publishing Group Ltd (unless otherwise stated in the text of the article). All rights reserved. No commercial use is permitted unless otherwise expressly granted.

\section{REFERENCES}

1 Hanash CR, Crosson JE. Emergency diagnosis and management of pediatric arrhythmias. J Emerg Trauma Shock 2010;3:251-60.

2 Canter C, Simpson K. Diagnosis and treatment of myocarditis in Children in the current era, 2014

3 Vignola PA, Aonuma K, Swaye PS, et al. Lymphocytic myocarditis presenting as unexplained ventricular arrhythmias: diagnosis with endomyocardial biopsy and response to immunosuppression. J Am Coll Cardio/ 1984;4:812-9.

4 Diercks DB, Shumaik GM, Harrigan RA, et al. Electrocardiographic manifestations: electrolyte abnormalities. J Emerg Med 2004;27:153-60.

5 Mattu A, Brady WJ, Robinson DA. Electrocardiographic manifestations of hyperkalemia. Am J Emerg Med 2000;18:721-9.

6 Marcolini EG, Keegan J, Injury BCEmerg Med Clin North Am 2015;33:519-27.

7 Behrle N, Dyke P, Dalabih A. Interventricular septal pseudoaneurysm after Blunt chest trauma in a 6 year Old: an illustrative case and review. Pediatr Emerg Care 2016.

8 Dowd MD, Krug S. Pediatric blunt cardiac injury: epidemiology, clinical features, and diagnosis. Pediatric Emergency Medicine Collaborative Research Committee: working Group on Blunt cardiac Injury. J Trauma 1996;40:61-7.

9 Baum VC. The patient with cardiac trauma. J Cardiothorac Vasc Anesth 2000;14:71-81.

10 Ottosen J, Guo A. Blunt Cardiac Injury The American Association for the surgery of Trauma. 2012 http://www.aast.org/blunt-cardiac-injury.

11 Healey MA, Brown R, Fleiszer D. Blunt cardiac injury: is this diagnosis necessary? J Trauma 1990;30:137-46.

12 Wisner DH, Reed WH, Riddick RS. Suspected myocardial contusion. triage and indications for monitoring. Ann Surg 1990;212:82-6.

13 Gunnar WP, Martin M, Smith RF, et al. The utility of cardiac evaluation in the hemodynamically stable patient with suspected myocardial contusion. Am Surg 1991;57:373-7.

14 Pasquale M, Fabian TC. Practice management guidelines for trauma from the Eastern Association for the surgery of Trauma. J Trauma 1998;44:941-56.

15 Norton MJ, Stanford GG, Weigelt JA. Early detection of myocardial contusion and its complications in patients with blunt trauma. Am J Surg 1990;160:577-82

16 Bock JS, Benitez RM. Blunt cardiac injury. Cardio/ Clin 2012;30:545-55.

17 Gonin J, de la Grandmaison GL, Durigon M, et al. Cardiac contusion and hemopericardium in the absence of external thoracic trauma: case report and review of the literature. Am J Forensic Med Patho/ 2009;30:373-5.

18 Huguet M, Tobon-Gomez C, Bijnens BH, et al. Cardiac injuries in blunt chest trauma. J Cardiovasc Magn Reson 2009;11:35. 
Copyright 2017 BMJ Publishing Group. All rights reserved. For permission to reuse any of this content visit http://group.bmj.com/group/rights-licensing/permissions.

BMJ Case Report Fellows may re-use this article for personal use and teaching without any further permission.

Become a Fellow of BMJ Case Reports today and you can:

- Submit as many cases as you like

- Enjoy fast sympathetic peer review and rapid publication of accepted articles

- Access all the published articles

- Re-use any of the published material for personal use and teaching without further permission

For information on Institutional Fellowships contact consortiasales@bmjgroup.com

Visit casereports.bmj.com for more articles like this and to become a Fellow 